\title{
Optimization and Application of Engineering Data Survey Based on Nonlinear Compensation Algorithm \\ Nanyou lu
}

\author{
Lanzhou Resources \& Environment Voc-Tech College 730021
}

Keywords: engineering data survey; information fusion; mapping; system design

\begin{abstract}
The effective engineering data survey algorithm is an important basis for engineering construction surveying, design, construction and management. In order to improve the survey accuracy of engineering data, the sensor survey method is researched, and the fusion detection and survey of engineering data is carried out. The traditional method used infrared and acoustic pulse detection method for frequency discrimination characteristic control and engineering data survey, when the engineering exploration stayed in a strong magnetic field, the engineering data survey curve achieved saturation, measuring performance is not good. An optimization method of engineering data survey algorithm is proposed based on nonlinear compensation control, and it is applied in the engineering surveying practice, quantitative analysis method is taken for realizing distributed engineering data survey nodes positioning and sensor information fusion, quantitative information fusion is taken in low dimensional state space, introducing end crosstalk sinusoidal pulse width modulation control of piezoelectric type permanent, and the magnetic detection circuit is designed, phase frequency characteristic survey method is presented. The system design is obtained. The adaptive noise cancellation method is taken for filtering, and the multi sensor data fusion filter structure model is designed. On the basis of the information distribution principle and nonlinear compensation, the fusion center is updated, and engineering data survey algorithm is improved. Simulation results show that, the engineering data survey algorithm is applied in the surveying and mapping of geographic Information calibration, the survey accuracy is improved, and the error is small, it has superior application performance in practice.
\end{abstract}

\section{Introduction}

Engineering Survey technology is one of the fundamental disciplines of surveying and mapping industry, all the surveying and mapping work in engineering construction are referred to as the engineering survey. In fact, it includes various survey works such as construction project survey, design, construction, installation, completion, monitoring and operation management and a series of engineering process service. It can be said, if there is no survey provides data and drawings for the construction of the project, any construction would not be processed and completed, thus, engineering data survey technology has important value and significance in the basic geographic information exploration and engineering construction fields. Generally speaking, engineering survey usually refers to the construction engineering survey and design, construction and management phase need use various survey theory, it is very important[1].

Traditional engineering survey technology service areas include building, water conservancy, transport, mining and other departments, and the basic contents have two parts as drawing and data sampling. The concept of modern engineering survey not only includes the static, dynamic geometry and physical quantity project determination, but also includes the analysis of the survey results, so the engineering data survey has important significance in the field of surveying and mapping. Accurate and effective engineering data survey algorithm design is an important basis for engineering construction surveying, design, construction and management[2], in order to improve the survey accuracy of engineering data, the sensor survey method is researched, the sensor measuring method, fusion engineering data and accurate detection and survey are researched, the traditional method used infrared and acoustic pulse detection method for frequency discrimination characteristic control and engineering data survey, when the engineering exploration stayed in a strong magnetic field, the engineering data survey curve achieved saturation, measuring 
performance is not good[3]. The reference [4] proposed the improved algorithm, a survey fusion algorithm for sensor engineering data energy management is proposed based on tracking differential delay and error compensation, but the algorithm is affected by noise is big, the accuracy is not high. In reference [5], a wireless tracking sensor engineering data analysis method is proposed based on priori information estimation, the condition has a good node transceiver channel equalization effect under the situation of priori information is unknown, but the algorithm need the decomposition and phase space rearrangement of signal time-frequency signal, calculation is large.

According to the problems as above, an optimization method of engineering data survey algorithm is proposed based on nonlinear compensation control, and it is applied in the engineering surveying practice, quantitative analysis method is taken for realizing distributed engineering data survey nodes positioning and sensor information fusion, quantitative information fusion is taken in low dimensional state space, introducing end crosstalk sinusoidal pulse width modulation control of piezoelectric type permanent, and the magnetic detection circuit is designed, phase frequency characteristic survey method is presented. The system design is obtained. The adaptive noise cancellation method is taken for filtering, and the multi sensor data fusion filter structure model is designed. On the basis of the information distribution principle and nonlinear compensation, the fusion center is updated, and engineering data survey algorithm is improved. Simulation results show that, the engineering data survey algorithm is applied in the surveying and mapping of geographic Information calibration, the survey accuracy is improved, and the error is small, it has superior application performance in future.

\section{Model construction of engineering data survey system and problem description}

\section{Multi sensor engineering data fusion model}

In order to optimize engineering data survey accuracy, it needs for sensor data fusion in survey project, accurate state estimation of the engineering data is realized, the base is to establish the multi sensor data fusion model. In the multi-sensor system, taking into account the adaptive transversal filter, in case the parameters are real, the survey error ${ }^{e}$ can be expressed as: $e(n)=d(n)-u^{T}(n) w(n)$, where, ${ }^{n}$ shows the $n$th iterative, $u$ is the input vector, $w$ is the weighted vector. The LMS estimates the squared error $e^{2}$ of ${ }^{i}$ time, the gradient representation takes the ${ }^{\nabla}$ as symbol. If the weight vector $W$ is recorded as ${ }^{W_{k}}$ at $k$ moment, in order to solve a weight vector make the mean square error be minimum, it can start from a set of initial value ${ }^{W_{0}}$, the gradient value is computed, and then select a new value ${ }^{W_{1}}$, let it be equal to initial value ${ }^{W_{0}}$, increment of the engineering data is proportional to the negative of the gradient. The next value is calculated ${ }^{W_{2}}$ from the gradient of $W_{1}$, using the same method to derive linear time invariant system, impulse response $H(n)$ in the signal processing is fixed. The weight $w_{1 k}$ is continuously adjusted to error $E\left[\varepsilon_{k}^{2}\right]$ in the adaptive processing, and it can reach the minimum value[6]. So, the response adaptive system can continuously reduce expectations and its own difference, the engineering data can be gauged, so that the system can gradually adapt to a given engineering data signal environment, survey model of engineering data can be expressed as follows:

$$
z_{k}^{i}=h_{k}^{i}\left(x_{k}, u_{k}\right)+v_{k}^{i}, i=1,2, \ldots, M
$$

The crosstalk sinusoidal pulse width modulation control is introduced, and the iezoelectric type permanent magnetic detection circuit is designed, phase frequency characteristic survey method is improved, the mathematical expression of engineering data vector is obtained:

Single input system:

$$
X_{k}=\left[x_{0}, x_{1}, x_{2}, \ldots . . x_{k}, \ldots . .\right]^{T}
$$

Multi input system:

$$
X_{k}=\left[x_{0 k}, x_{1 k}, x_{2 k}, \ldots . . x_{l k}, \ldots . .\right]^{T}
$$

According to the input of engineering data as above, we can get the relationship between system output and input as: 
Output of single input system:

$$
\begin{aligned}
& \qquad y_{k}=\sum_{t=0}^{l} w_{l k} x_{k-l} \\
& \text { Output of multi input system: } y_{k}=\sum_{t=0}^{l} w_{l k} x_{l k}
\end{aligned}
$$

In the formula, $i(i=1,2, \ldots, M)$ is the parameter corresponding to the sensor (e.g. $z_{k}^{i}$ shows survey vector vector in the moment sensing), $v_{k}^{i}$ is the sensor $i$ survey noise, $E\left[v_{k}^{i}\left(v_{j}^{i}\right)^{T}\right]=R_{k}^{i} \delta_{k j}, E\left[w_{k}\left(v_{j}^{i}\right)^{T}\right]=0$; Consider the sensor network sensor with $\mathrm{N}$ nodes, the system output $y_{k}$ and the expected system response $d_{k}$ has error $\varepsilon_{k}$, thus obtained the error expression of system $\varepsilon_{k}=d_{k}-y_{k}=d_{k}-X_{k}{ }^{T} W_{k}=d_{k}-W_{k}^{T} X_{k}$. The expression for the mean square error is:

$$
E\left[\varepsilon_{k}{ }^{2}\right]=E\left[d_{k}{ }^{2}\right]+W_{k}^{T} E\left[X_{k} X_{k}^{T}\right] W_{k}-2 E\left[d_{k} X_{k}{ }^{T}\right] W_{k}
$$

$\mathrm{R}$ is defined as the matrix:

$$
R=E\left[X_{k} X_{k}^{T}\right]=E\left[\begin{array}{cccc}
x_{0 k}^{2} & x_{0 k} x_{1 k} & \cdots & x_{0 k} x_{L k} \\
x_{1 k} x_{0 k} & x_{1 k}^{2} & \cdots & x_{1 k} x_{L k} \\
\vdots & \vdots & \ddots & \vdots \\
x_{L k} x_{0 k} & x_{L k} x_{1 k} & \cdots & x_{L k}^{2}
\end{array}\right]
$$

Among them, $\mathbf{x}(k) \in \mathbf{R}^{n \times 1}$ is the target state, $\mathbf{A}(k) \in \mathbf{R}^{n \times n}$ is the state transition matrix, the multi sensor network model and engineering data sensor networks are designed based on the tracking system, engineering data of sensor nodes are fused, then, need to achieve a filter for engineering survey data and fusion, the multi sensors data fusion filter structure model is shown in Figure 1.

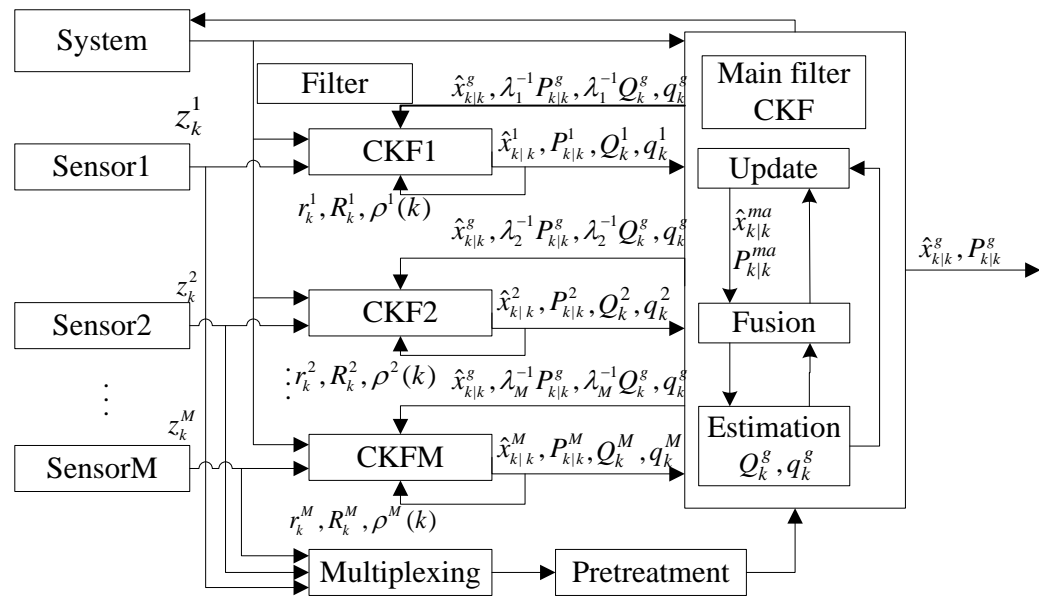

Figure 1 Multi sensor data fusion filter structure model of engineering survey data

In Figure 1, $g$ represents the global parameters, $\lambda_{i}(i=1,2, \ldots, M, m a)$ are the information distribution coefficients, the amount of state estimation information can be expressed as $P^{-1}$, the state equation information can be expressed as $Q^{-1}$. Multi sensor data fusion of multiple information feedback process exists combinational filter structure, each sub filter adopts adaptive information distribution method for state estimation optimization, sensor data fusion is realized, the design also needs to further reduce the computation cost in real-time fusion center, in this paper, the strong tracking filter compensation is taken as the foundation.

\section{Analysis of nonlinear compensation algorithm for engineering data survey}

An optimization method of engineering data survey algorithm is proposed based on nonlinear compensation control, and it is applied in the engineering surveying practice, quantitative analysis method is taken for realizing distributed engineering data survey nodes positioning and sensor information fusion, basic principle of nonlinear compensation control is expressed as follows: the number of reference nodes ${ }^{o_{i}}$ is $L(L \geq 3)$, randomly selected 6beacon nodes, if $K<6$, final coordinate generation node of nonlinear compensation average value is $\left(x_{i, t+1}, y_{i, t+1}\right)$, , the formula for calculating: 


$$
\left\{\begin{array}{l}
x_{i, t+1}=\left(x_{i, t}+x_{i, t+1}^{\prime}\right) / 2 \\
y_{i, t+1}=\left(y_{i, t}+y_{i, t+1}^{\prime}\right) / 2
\end{array}\right.
$$

Based on multi sensor network nonlinear compensation control problems, because the deviation of engineering data of survey bandwidth limit control system is $f(l), \Delta f(l)$ is the deviation of transform coefficients, it is used to describe the entire emission probe impedance into perceptual error. Use the following formula to describe the phase discriminator circuit characteristic survey control rule:

$$
\eta=\frac{B_{j}-\sqrt{C_{j}-D_{j}^{2}}}{E_{j} \cdot F_{j}+1}
$$

In the formula, ${ }^{B_{j}}$ is a nonlinear compensation control survey system bias variable value in far end crosstalk, ${ }^{C_{j}}$ is the deviation coefficient of variation values, ${ }^{D_{j}}$ is the far end crosstalk compensation vector value of the variable ${ }^{L_{q}}$ in the phase offset detection circuit, ${ }^{E_{j}}$ is the variable data in the survey process, $F_{j}$ is the error compensation parameter. Thus, the quantitative information fusion is taken in low dimensional state space, introducing end crosstalk sinusoidal pulse width modulation control of piezoelectric type permanent, and the magnetic detection circuit is designed, the algorithm is improved.

\section{Design and realization of improved engineering data survey system}

In order to improve the survey accuracy of engineering data, the sensor survey method is researched, and the fusion detection and survey of engineering data is carried out. The traditional method used infrared and acoustic pulse detection method for frequency discrimination characteristic control and engineering data survey, when the engineering exploration stayed in a strong magnetic field, the engineering data survey curve achieved saturation, measuring performance is not good. An optimization method of engineering data survey algorithm is proposed based on nonlinear compensation control, considering the tracking process by object dynamic sensor network system with $\mathrm{N}$ sensor nodes, the survey noise $\mathbf{u}_{i}(k) \in \mathbf{R}^{p \times 1}$, the process noise $\mathbf{w}(k)$ to $\mathbf{u}_{i}(k)$ has mutual correlation, ${ }^{i=1,2, \cdots, N}$, dimensional survey equation can be expressed as:

$$
\mathbf{M}(k)=\mathbf{H}(k) \mathbf{x}(k)+\mathbf{V}(k) \quad(10)
$$

Fusion algorithm is designed, obtain node information, and it is quantified, fusion center receives the original survey information, and the quantization noise can be filtered, can be expressed as the original surveys and the quantization noise form, as:

$$
\mathbf{m}_{i}(k)=\mathbf{z}_{i}(k)+\mathbf{q}_{i}(k)=\mathbf{H}_{i}(k) \mathbf{x}(k)+\mathbf{u}_{i}(k)+\mathbf{q}_{i}(k)=\mathbf{H}_{i}(k) \mathbf{x}(k)+\mathbf{v}_{i}(k)
$$

Among them, given bandwidth constraints and quantization threshold, the variance of the quantization noise meets:

$$
\mathbf{R}_{q}^{i}(k)=E\left[\mathbf{q}_{i}(k) \mathbf{q}_{i}^{T}(k)\right] \leq \operatorname{diag}\left\{\Delta_{i}^{2}(k, 1) / 4, \Delta_{i}^{2}(k, 2) / 4, \cdots, \Delta_{i}^{2}(k, p) / 4\right\}=\overline{\mathbf{R}}_{q}^{i}(k)
$$

The information state estimation is $\hat{\mathbf{y}}(k \mid k)$, and the recursive formula of information matrix $\mathbf{Y}(k \mid k)$ is:

$$
\begin{gathered}
\mathbf{Y}(k \mid k-1)=\left\{\mathbf{I}-\mathbf{F}(k-1)\left[\mathbf{F}(k-1)+\breve{\mathbf{Q}}^{-1}(k-1)\right]^{-1}\right\} \times \mathbf{F}(k-1) \\
\mathbf{Y}(k \mid k)=\mathbf{Y}(k \mid k-1)+\breve{\mathbf{H}}^{T}(k) \mathbf{R}^{-1}(k) \breve{H}(k)
\end{gathered}
$$

Set $I_{k}=\left\{u_{i}, z_{i}\right\}_{i=1}^{k}$ represents a collection of sensor nodes in all the control input and survey, by changing the tracking signal power dynamically, we obtain the adaptive iteration step, and the survey engineering data should be selected as:

$$
\begin{gathered}
\mu_{1}(k)=(1-\beta) /\left[\sigma_{x 1}{ }^{2}(k)\right] \\
\sigma_{x 1}{ }^{2}(k)=\beta \sigma_{x 1}{ }^{2}(k-1)+(1-\beta) x_{1}^{2}(k),(0<\beta<1)
\end{gathered}
$$

$X_{i, k+1 \mid k}$ is taken in the nonlinear survey equation, the state vector of node location is:

$$
Z_{i, k+1 \mid k}^{*}=h_{k+1}\left(X_{i, k+1 \mid k}, u_{k}\right)+r_{k}, i=1,2, \ldots, m
$$

Then, the engineering data survey value can be estimated as: 


$$
\hat{z}_{k+1 \mid k}=m^{-1} \sum_{i=1}^{m} Z_{i, k+1 \mid k}^{*}+r_{k}
$$

The survey results obtained in this paper is the global optimal unbiased, it can be effectively applied in mapping and geography environment.

\section{Simulation experiments and results analysis}

In order to test the algorithms proposed in this paper, the performance of fusion survey in the realization of engineering data is simulated, simulation experiment is taken, and the hardware system of simulation experimental platform is described as: model Dell 2210b, the processor for the Intel Core2 Duo1.80 GHz, $1 \mathrm{G}$ of ram, clocked at DDR2 667, the simulation software is Matlab 7. Construct the system of engineering data survey system based on nonlinear compensation control and wireless sensor network, the maximum distance between the sensor nodes $R_{\max }$ is $1000 \mathrm{~m}, R_{\min }$ is $200 \mathrm{~m}$. the sensor nodes transmit signals between the length of 16QAM is 12000, the strong tracking filter tap coefficient is 11 , the bandwidth is $0.5 \mathrm{~s}$, the relative bandwidth is 0.8 . Based on the simulation environment, the measuring equation for each sensor: $\mathbf{z}_{i}(k)=\left[\begin{array}{ll}1 & 0\end{array}\right] \mathbf{x}(k)+\mathbf{u}_{i}(k), \quad i=1,2,3$.

The ranging errors of each sensor are respectively $2 \mathrm{~m}, 1.5 \mathrm{~m}$ and $1.5 \mathrm{~m}$. Signals are collected by multiple sensors, this design data fusion model is used, and the engineering data survey is realized, the time-frequency space and the amplitude frequency space of engineering data are shown in Figure 2.

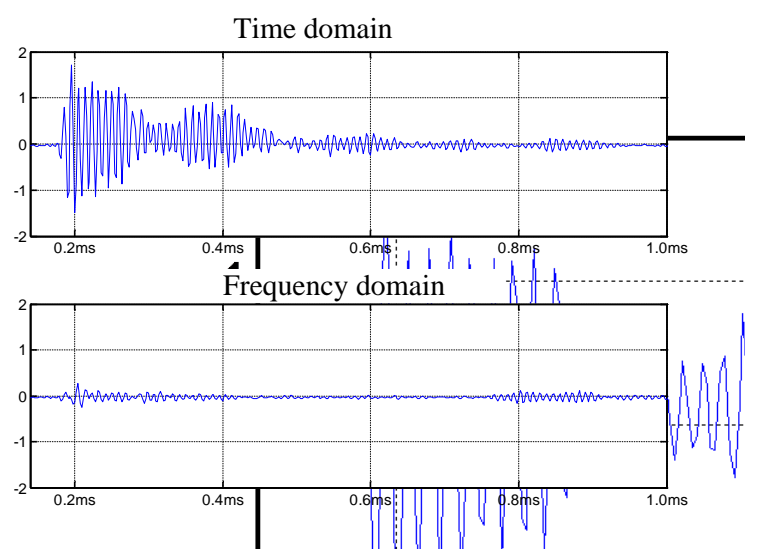

Figure 2. Time-frequency space and the amplitude frequency space of engineering data

Figure 2 gives the data fusion model, it is takne as the basis, based on nonlinear compensation control, the sample data is inputed into the engineering filtering system, noise suppression is completed, multi sensor data fusion filter structure model is designed. On the basis of the information distribution principle and nonlinear compensation, the fusion center is updated, the accurate positioning of engineering data survey are shown in Figure 3.

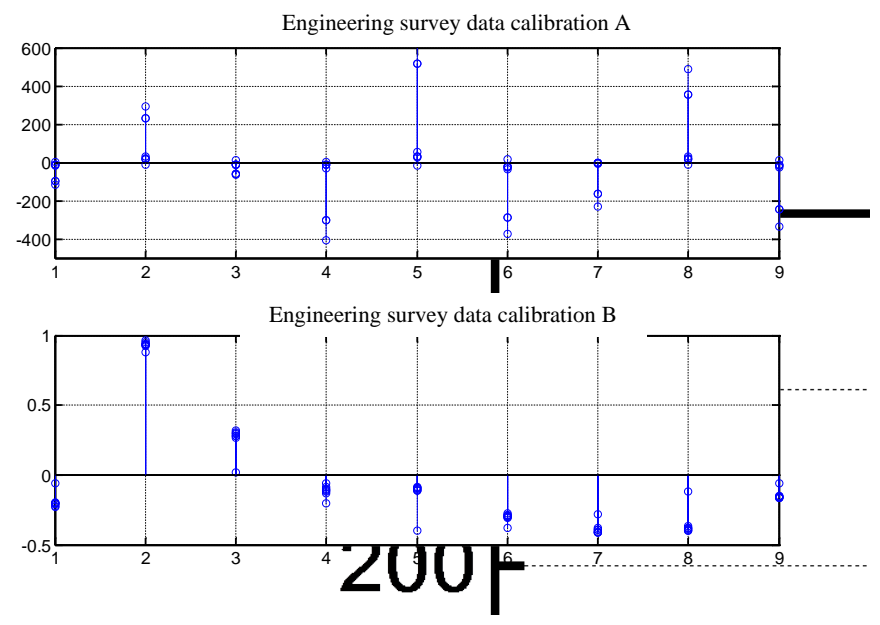

Figure 3. Engineering survey point positioning simulation results

Finally, optimization method of engineering data measuremen proposed in this paper is applied 
in the geographical mapping, geographic location for information calibration is realized, and the survey accuracy is taken as the evaluation standard. The comparison results of this algorithm and the traditional algorithm are shown in Figure 4, it can be seen from the diagram, the proposed algorithm can effectively improve the accuracy of surveying and mapping, the precision of the calibration points is improved by $34 \% \sim 53 \%$, it shows the better performance of surveying and mapping.

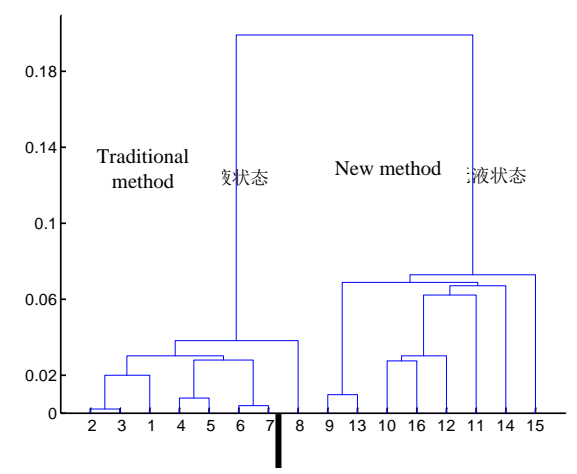

Figure 4. Accuracy comparison of engineering data mapping and surveying

\section{Conclusions}

In this paper, an optimization method of engineering data survey algorithm is proposed based on nonlinear compensation control, and it is applied in the engineering surveying practice. On the basis of the information distribution principle and nonlinear compensation, the fusion center is updated, and engineering data survey algorithm is improved. Simulation results show that, the proposed algorithm can effectively improve the accuracy of surveying and mapping, the precision of the calibration points is improved by 34\% 53\%, it shows the better performance of surveying and mapping, and the error is small, so it has superior application performance in practice.

\section{References}

[1] MANSOURI M, ILHAM O, SNOUSSI H, et al.. Adaptive quantized target tracking in wireless sensor networks [J]. Wireless Networks, 2011, 17(7):1625- 1639.

[2] ZHOU Yan, LI Jian-xun, WANG Dong-li. Target tracking in wireless sensor networks using adaptive survey quantization [J]. Science China Information Sciences, 2012, 55(4):827-838.

[3] XU Jian, LI Jian-xun, XU Sheng. Data fusion for target tracking in wireless sensor networks using quantized innovations and Kalman filtering [J]. Science China Information Sciences, 2012, 55(3):530-544.

[4] MASAZADE E, NIU Rui-xin, VARSHNEY P. Dynamic bit allocation for object tracking in wireless sensor networks [J]. IEEE Transactions on Signal Processing, 2012, 60(10):5048-5063.

[5] Shen Yuan. Research on Intrusion Detection System Neural Networks and Principal Component Analysis[J]. Bulletin of Science and Technology. 2013; 29(6): 32-34.

[6] JIN Xiao-yan, ZHOU Xi-yuan, ZHANG Wan-lin. Modulation Recognition Using Adaptive MCMC in Multipath Fading Channel, Modulation R ecognition Using Adaptive MCMC in Multipath Fading Channel[J]. Journal of Beijing University of Posts and Telecommunications, 2014, 37(1): 31-34. 